\title{
A THEOREM ON DIFFERENCE POLYNOMIALS
}

\author{
RICHARD M. COHN
}

We shall prove the following analogue of a theorem of Kronecker's: Let $\mathcal{F}$ be a difference field ${ }^{1}$ containing an element $t$ which is distinct from its transforms of any order. Every perfect ideal in the ring $\mathcal{F}\left[y_{1}, \cdots, y_{n}\right]$ has a basis consisting of $n+1$ difference polynomials.

The corresponding theorem for differential polynomials was proved by J. F. Ritt. ${ }^{2}$ We shall follow in all but details a brief and elegant proof of the theorem for differential equations which he has given recently. ${ }^{3}$

Let $\Lambda$ be a perfect ideal in $\mathcal{F}\left[y_{1}, \cdots, y_{n}\right]$. We know that $\Lambda$ has a finite basis $A_{1}, \cdots, A_{r}$. We adjoin to $\mathcal{F}\left[y_{1}, \cdots, y_{n}\right]$ the $(n+1) r$ additional variables $u_{i j}, i=1, \cdots, n+1 ; j=1, \cdots, r$, and consider the polynomials ${ }^{5}$

$$
L_{i}=\sum_{j=1}^{r} u_{i j} A_{j}, \quad i=1, \cdots, n+1 .
$$

We shall show that there exists a nonzero polynomial $H$ in the $u_{i j}$ only, which vanishes for all solutions of the system (1) which do not annul every $A_{i}$. Our theorem will follow immediately from the existence of $H$. For I showed in my Dissertation ${ }^{6}$ that the presence of $t$ in $\mathcal{F}$ implies that we may assign to the $u_{i j}$ values $\alpha_{i j}$ in $\mathcal{F}$ which do not annul $H$. Let $L_{i}$ become $M_{i}, i=1, \cdots, n+1$, when the $u_{i j}$ are

Received by the editors April 29, 1948.

${ }^{1}$ For a definition of the terms used in the statement of this theorem the reader is referred to J. F. Ritt and H. W. Raudenbush, Ideal theory and algebraic difference equations, Trans. Amer. Math. Soc. vol. 46 (1939) pp. 445-452. The reader's attention is also called to the paper Complete difference ideals by J. F. Ritt, Amer. J. Math. vol. 63 (1941) p. 681, in which the definition of the term "difference ideal" is modified. It is the latter definition which is now in use. The symbol $\mathcal{f}\left[y_{1}, \cdots, y_{n}\right]$ denotes the ring of all difference polynomials in the unknowns $y_{1}, \cdots, y_{n}$, whose coefficients lie in the difference field $\mathcal{F}$.

${ }^{2} \mathrm{~J}$. F. Ritt, Differential equations from the algebraic standpoint, Amer. Math. Soc. Colloquium Publications, vol. 14, pp. 50-58.

${ }^{3}$ To appear in the forthcoming revised edition of Differential equations from the algebraic standpoint. This shorter proof does not furnish all details of the theorem established in the paper mentioned in footnote 2.

${ }^{4}$ This is one of the principal results of the paper of Ritt and Raudenbush referred to in footnote 1.

${ }^{5}$ We use the term "polynomial" as an abbreviation for "difference polynomial."

${ }^{6}$ Manifolds of difference polynomials, Trans. Amer. Math. Soc. vol. 64 (1948) pp. 133-172. This paper is referred to below as M.D.P. 
thus specialized. Then every solution of the system $M_{i}=0$ can be extended to a solution of (1) by letting $u_{i j}=\alpha_{i j}, i=1, \cdots, n+1$, $j=1, \cdots, r$. Since $H$ does not vanish it follows that the $A_{i}$ are all zero. Thus the $M_{i}$ are a basis for $\Lambda$ containing $n+1$ polynomials.

It remains only to prove the existence of $H$. We make successive transformally transcendental adjunctions ${ }^{7}$ to $\mathcal{F}$ of elements $v_{i}$, $i=1, \cdots, n ; w_{i j}, i=1, \cdots, n+1, j=2, \cdots, r$. We let each $y_{i}=v_{i}$, and each $u_{i j}=w_{i j}, j>1$. The $u_{i 1}$ may then be computed from the equations $L_{i}=0$. Let $\Sigma$ be the reflexive ${ }^{8}$ prime ideal consisting of all polynomials in the $u_{i j}$ and $y_{i}$ which are annulled by this solution.

$\Sigma$ contains no difference polynomial in the $y_{i}, i=1, \cdots, n$, and $u_{i j}, j>1$, alone; for no such polynomial is annulled by the solution just described. Each $L_{i}$ is in $\Sigma$, so that, for each $i$ from 1 to $n+1, \Sigma$ contains a polynomial in $u_{i 1}$ which is free of the other $u_{j 1}$. Thus the $u_{i j}, j>1$, and $y_{i}$ may be chosen as a set of arbitrary unknowns for $\Sigma$. The $u_{i j}$ are more numerous than the arbitrary unknowns so that, by Theorem IV of M.D.P., $\Sigma$ contains a nonzero polynomial $H_{1}$ in the $u_{i j}$ only.

It is evident that the $L_{i}$ constitute a basic set ${ }^{9}$ of $\Sigma$ when we choose for the arbitrary unknowns the set indicated above. The remainder of $H_{1}$ with respect to this set is zero. It follows, since $A_{1}$ is the initial of each $L_{i}$, that there exists a product $P$ of powers of transforms of $A_{1}$ such that $P H_{1}$ is a linear combination of the $L_{i}$ and their transforms. Then $H_{1}$ vanishes for all solutions of (1) which do not annul $A_{1}$.

We may show in the same way that there exist nonzero polynomials $H_{i}, i=2, \cdots, r$, in the $u_{i j}$ only, such that $H_{k}$ vanishes for every solution of (1) which does not annul $A_{k}$. Then $H=\prod_{i=1}^{r} H_{i}$ is the polynomial whose existence we wished to prove. The theorem is established.

It is perhaps surprising that $n+1$ polynomials should suffice for a basis of a perfect difference ideal in $n$ unknowns, when it is known that many more polynomials may be required in a basic set. As an

7 This is to say that the $v_{i}$ and $w_{i j}$ annul no difference polynomial with coefficients in F. See M.D.P., §6.

${ }^{8} \mathrm{~A}$ difference ideal is called reflexive if the inclusion in the ideal of the transform $C_{1}$ of a difference polynomial $C$ implies that $C$ itself is in the ideal. See the paper Complete difference ideals referred to in footnote 1.

${ }^{9}$ Basic sets of systems of difference polynomials are defined by J. F. Ritt and J. L. Doob in their paper, Systems of algebraic difference equations, Amer. J. Math. vol. 55 (1933) pp. 506-508. It should be noted that the definition of ascending sets, which is preliminary to that of basic sets, is substantially different from that of ascending sets of differential polynomials. 
illustration of this phenomenon we give the following example of an ideal with a basis which is a subset of its basic set. ${ }^{10}$

Let $\mathcal{F}$ be the field of all rational functions of $x$ with transforming defined as the operation of replacing $x$ by $x+1$. We consider the polynomials

$$
y_{1}-y, \quad z^{2}-y, \quad z_{1}-z .
$$

Using Theorem IX of M.D.P. one shows easily that (2) is a basic set of a prime reflexive ideal $\Lambda$ with coefficients in $\mathcal{F}$. Since the initials of the polynomials of (2) are unity, it follows that it is a basis for $\Lambda$. But the equations $z_{1}-z=0, z^{2}-y=0$, imply that $y_{1}=z_{1}^{2}=z^{2}=y$; so that any solution of these equations is also a solution of $y_{1}-y=0$. Thus $z^{2}-y, z_{1}-z$ is itself a basis for $\Lambda$.

RUTGERS UNIVERSITY

${ }^{10}$ This example emerged during a conversation of the author with E. R. Kolchin.

\section{INVERSIVE DIFFERENCE FIELDS}

\section{RICHARD M. COHN}

It may happen that the functions of an analytic difference field admit not only the operation of replacing $z$ by $z+1$, but also its inverse. Since these two operations have essentially the same properties it is to be expected that to each theorem concerning difference equations there will be a corresponding theorem, valid in fields of the type we have just described, in which the rôles of the highest and lowest transforms of the unknowns are interchanged. For example, J. F. Ritt has shown ${ }^{1}$ that the number of ordinary manifolds of a firstorder difference polynomial in the unknown $y$ does not exceed its degree in $y_{1}$; and he observes that in fields where the inverse substitution is always possible, the number of ordinary manifolds is also limited by the degree in $y$.

The study of abstract difference fields enables us to apply this principle in every case; for, as we shall show, every abstract difference field can be extended to a difference field in which there exists, for every element $h$ of the field, an element $g$ such that $h$ is the trans-

Received by the editors April 29, 1948.

${ }^{1}$ J. F. Ritt, Algebraic difference equations, Bull. Amer. Math. Soc. vol. 40 (1934) pp. 303-308. 